\title{
Potential regulatory role of circular RNA in idiopathic pulmonary fibrosis
}

\author{
RONGRONG LI ${ }^{*}$, YOULEI WANG ${ }^{2 *}$, XIAODONG SONG ${ }^{3 *}$, WENJING SUN $^{4}$, \\ JINJIN ZHANG ${ }^{3}$, YUXIA LIU ${ }^{1}$, HONGBO LI ${ }^{1}, \mathrm{CHAO} \mathrm{MENG}^{3}$, \\ JIE ZHANG ${ }^{3}$, QINGYIN ZHENG ${ }^{2}$ and CHANGJUN LV ${ }^{1}$ \\ ${ }^{1}$ Department of Respiratory Medicine, Affiliated Hospital of Binzhou Medical University, Binzhou, \\ Shandong 256602; ${ }^{2}$ School of Special Education, Binzhou Medical University; ${ }^{3}$ School of Pharmacy, \\ Binzhou Medical University, Yantai, Shandong 264003; ${ }^{4}$ School of Life Sciences, \\ Ludong University, Yantai, Shandong 264025, P.R. China
}

Received January 26, 2018; Accepted August 2, 2018

DOI: $10.3892 /$ ijmm.2018.3892

\begin{abstract}
Idiopathic pulmonary fibrosis (IPF) is a chronic, progressive type of interstitial pneumonia with unknown causes, poor prognosis and no effective therapy available. Circular RNAs (circRNAs), which serve as potential therapeutic targets and diagnostic biomarkers for certain diseases, represent a recent hotspot in the field of RNA research. In the present study, a total of 67 significantly dysregulated circRNAs were identified in the plasma of IPF patients by using a circRNA microarray. Among these circRNAs, 38 were upregulated, whereas 29 were downregulated. Further validation of the results by polymerase chain reaction analysis indicated that Homo sapiens (hsa)_circRNA_100906,hsa_circRNA_102100 and hsa_circRNA_102348 were significantly upregulated, whereas hsa_circRNA_101225, hsa_circRNA_104780 and hsa_circRNA_101242 were downregulated in plasma samples of IPF patients compared with those in samples from healthy controls. The majority of differentially expressed circRNAs were generated from exonic regions. The host genes of the differentially expressed circRNAs were involved in the regulation of the cell cycle, adherens junctions and RNA transport. The competing endogenous RNA (ceRNA) network of the circRNAs/micro(mi)RNAs/mRNAs indicated
\end{abstract}

Correspondence to: Professor Jinjin Zhang, School of Pharmacy, Binzhou Medical University, 346 Guanhai Road, Laishan, Yantai, Shandong 264003, P.R. China

E-mail: jjinzhang@126.com

Professor Changjun Lv, Department of Respiratory Medicine, Affiliated Hospital of Binzhou Medical University, 661 Huanghe 2nd Road, Binzhou, Shandong 256603, P.R. China

E-mail: lucky_lcj@sina.com

*Contributed equally

Key words: idiopathic pulmonary fibrosis, circular RNA, competing endogenous RNA, microRNA, host gene that circRNA-protected mRNA participated in transforming growth factor- $\beta 1$, hypoxia-inducible factor- 1 , Wnt, Janus kinase, Rho-associated protein kinase, vascular endothelial growth factor, mitogen-activated protein kinase, Hedgehog and nuclear factor $\kappa \mathrm{B}$ signalling pathways or functioned as biomarkers for pulmonary fibrosis. Furthermore, luciferase reporter assays confirmed that hsa_circRNA_100906 and hsa_circRNA_102348 directly interact with miR-324-5p and miR-630, respectively, which were downregulated in IPF patients. The present study provided a novel avenue for exploring the underlying molecular mechanisms of IPF disease.

\section{Introduction}

Idiopathic pulmonary fibrosis (IPF) is a chronic, progressive, and usually lethal disorder with an unknown etiology, a median survival time of 3-5 years and a mortality rate exceeding those of numerous cancer types $(1,2)$. Current pathogenetic theories suggest that IPF is a result of an aberrant wound healing response and characterized by injured alveolar epithelium, formation of fibroblast/myofibroblast foci and accumulation of extracellular matrix (ECM) (3). Despite the marked increase in research efforts, the exact mechanisms involved in the initiation, maintenance and progression of fibrosis remain largely elusive (4). In recent years, RNA-based regulation, including that involving micro (mi)RNA, long non-coding (nc)RNA or circular (circ)RNA, has been identified in fibrotic disease (5-7).

circRNA represents a novel class of endogenous ncRNA, which is observed in a wide variety of organisms $(8,9)$. Unlike linear RNA, circRNA demonstrates unusual stability due to its unique covalent loop structure with neither 5' to $3^{\prime}$ polarity nor a polyadenylated tail (10). With the rapid development of high-throughput sequencing technology, circRNA has been identified to be ubiquitously expressed in various tissues and cells types (11). Its biogenesis is influenced by cis elements and transfactors, including Alu repeats, reverse complementary matches and RNA-binding protein (12). Furthermore, tissue, cell type or developmental stage-specific expression 
patterns imply that circRNA has important biological functions $(13,14)$. Emerging evidence demonstrates that circRNAs serve as gene regulators in mammals, particularly through functioning as miRNA sponges or mRNA translation templates, facilitating transcription of their host genes by directly associating with RNA polymerase II or forming platforms for protein interactions $(15,16)$. circRNAs have crucial roles in cell homeostasis and are closely correlated with the clinical and pathological features of various human diseases, including atherosclerosis, neurological disorders, fibrosis and cancer; thus, circRNAs have potential as novel clinical diagnostic and prognostic biomarkers $(17,18)$. However, neither the formation mechanism nor the cellular function of circRNA has been completely understood in IPF. In the present study, the dysregulated circRNAs in IPF were identified through microarrays and the potential circRNA-associated competing endogenous RNA (ceRNA) network was constructed with the aim to provide novel biomarkers for IPF diagnosis and pathogenesis.

\section{Materials and methods}

Patients and clinical samples. Patients admitted to the Affiliated Hospital of Binzhou Medical University (Binzhou, China) who were diagnosed with IPF between October 2015 and June $2017(n=10)$ through combined clinical, radiological and pathological examination based on the American Thoracic Society/European Respiratory Society consensus criteria (19) were included in the present study. Normal controls from healthy volunteers were matched corresponding to the IPF patients' sex and age. The healthy non-smoking volunteers were recruited from individuals undergoing a physical examination at the hospital over the same time period. Individuals with respiratory and rheumatic immune diseases were excluded from the study. Fresh peripheral blood samples were collected in EDTA-containing tubes and then centrifuged $(1,000 \times \mathrm{g}$ for $10 \mathrm{~min}$ at room temperature) to isolate the plasma.

RNA extraction and characterization. The total RNA of each sample was isolated using TRIzol LS reagent (Invitrogen; Thermo Fisher Scientific, Inc., Waltham, MA, USA) according to the manufacturer's instructions. The RNA quantity and quality were determined using a NanoDrop ND-1000 instrument (Thermo Fisher Scientific, Inc.). A 260/280 nm absorbance ratio between 1.8 and 2.1 , or a $260 / 230 \mathrm{~nm}$ absorbance ratio of $>1.8$ were acceptable. RNA integrity and genomic DNA contamination were assessed by standard denaturing agarose gel electrophoresis after RNA extraction and prior to sample labelling. DNA contamination was ruled out by the absence of a high molecular-weight smear or band migration above the 28S ribosomal RNA band. RNA integrity was confirmed by the absence of RNA degradation, which was reflected by smearing of ribosomal RNA bands.

Microarray hybridization. Arraystar Inc. (Rockville, MD, USA) developed the world's first commercial circRNA chip for systematically analysing the expression of circRNA in different physiological and pathological conditions. The Arraystar Human circRNA Array (8x15 K; Arraystar Inc.) analysis was performed by Kangchen Corp. (Shanghai, China) in 3 pairs of IPF samples and normal control samples. In brief, isolated total RNA was treated with RNase R (Epicenter, Madison, WI, USA) to remove linear RNA. Subsequently, the samples were amplified and transcribed into fluorescent complementary (c)RNA by utilizing a random primer method according to the Arraystar Super RNA Labeling protocol. Subsequently, the labelled cRNA was purified using an RNeasy Mini Kit (Qiagen, Hilden, Germany). The concentration and specific activity of the labelled cRNA (pmol Cy3/ $\mu \mathrm{g}$ cRNA) was measured using a NanoDrop ND-1000. A total of $1 \mu \mathrm{g}$ of each labelled cRNA was then fragmented by adding $5 \mu \mathrm{l}$ of $10 \mathrm{X}$ blocking agent and $1 \mu \mathrm{l}$ of $25 \mathrm{X}$ fragmentation buffer. The mixture was then heated at $60^{\circ} \mathrm{C}$ for $30 \mathrm{~min}$, and $25 \mu 12 \mathrm{X}$ hybridization buffer was then added to dilute the labelled cRNA. Subsequently, $50 \mu \mathrm{l}$ of the hybridization solution was dispensed into the gasket slide and assembled onto the Arraystar circRNA expression microarray slide. The slides were incubated for $17 \mathrm{~h}$ at $65^{\circ} \mathrm{C}$ in an Agilent hybridization oven (Agilent Technologies, Inc., Santa Clara, CA, USA). The slides were washed and the hybridized arrays were scanned using an Agilent G2505C scanner (Agilent Technologies, Inc.). The scanned images were imported into the Agilent feature extraction software (version 11.0.1.1) to extract raw data (Agilent Technologies, Inc.).

Microarray data analysis. Quantile normalization of raw data and the subsequent data processing were performed using the $\mathrm{R}$ limma package (bioconductor. org/packages/release/bioc/html/limma.html). After quantile normalization of raw data, low-intensity filtering was performed and circRNA with a minimum of 1 out of 6 samples with flags in 'present' or 'marginal' ('all target values') were retained for further analyses. In the comparison of the two groups, namely disease vs. normal controls, the 'fold change' (the ratio of group average values) among groups was computed for each circRNA. Statistical significance of the difference was estimated via a Student's t-test. circRNA with an absolute value of the fold change $\geq 1.5$ and $P$-values of $\leq 0.05$ were considered to indicate significantly different expression. The final outputs were filtered and the differentially expressed circRNA was ranked based on fold change and P-value, among others, by using the 'Data/Sort' and 'Filter' functions of Microsoft Excel2013 (Microsoft Corp., Redmond, WA, USA). The dataset was deposited in the Gene Expression Omnibus database (https://www.ncbi.nlm.nih.gov/geo/) with the accession no. GSE102660.

Reverse transcription (RT). cDNA for circRNA and mRNA was generated using the Super Script ${ }^{\mathrm{TM}}$ III First-Strand Synthesis System (Invitrogen; Thermo Fisher Scientific, Inc.) following the manufacturer's protocol. In brief, $3 \mu \mathrm{g}$ total RNA, $1 \mu \mathrm{l}$ random primer (N9), $4 \mu \mathrm{l} 5 \mathrm{X}$ first-strand buffer, $1.6 \mu \mathrm{l}$ nucleotide mix, $0.3 \mu \mathrm{l}$ RNase inhibitor, $1 \mu \mathrm{l}$ dithiothreitol and $0.2 \mu 1$ Super Script ${ }^{\mathrm{TM}}$ III reverse transcriptase were added to the system, which was then incubated at $50^{\circ} \mathrm{C}$ for $1 \mathrm{~h}$. The RT reaction and no-template control were performed simultaneously. RT of miRNAs was performed using the Bulge-Loop miRNA primer set according to the manufacturer's protocol (RT miR-324-5p primer, cat. no. ssD809230302; RT miR-630 
primer, cat. no. ssD809230591; Guangzhou RiboBio Co., Ltd. (Guangzhou, China).

Quantitative polymerase chain reaction ( $q P C R)$. The expression levels of circRNA and miRNA were evaluated via qPCR using 2X PCR master mix (Arraystar Inc.) and SYBR Green Master Mix (Roche Diagnostics, Basel, Switzerland), respectively, according to the manufacturer's protocol. PCR for circRNA was performed in a $10-\mu 1$ reaction volume, including $2 \mu \mathrm{l} \mathrm{cDNA,} 5 \mu \mathrm{l}$ 2X Master Mix, $0.5 \mu \mathrm{l}$ forward primer $(10 \mu \mathrm{M}), 0.5 \mu \mathrm{l}$ reverse primer $(10 \mu \mathrm{M})$ and $2 \mu \mathrm{l}$ double-distilled water. The reaction was set at $95^{\circ} \mathrm{C}$ for $10 \mathrm{~min}$ for pre-denaturation, followed by 40 cycles of $95^{\circ} \mathrm{C}$ for $10 \mathrm{sec}$ and $60^{\circ} \mathrm{C}$ for $60 \mathrm{sec}$. GAPDH was used for template normalization of circRNA (20). PCR for miR-324-5p and miR-630 entailed initiation at $95^{\circ} \mathrm{C}$ for $20 \mathrm{sec}$, followed by 30 cycles at $95^{\circ} \mathrm{C}$ for $10 \mathrm{sec}, 60^{\circ} \mathrm{C}$ for $20 \mathrm{sec}$ and $72^{\circ} \mathrm{C}$ for $10 \mathrm{sec}$. PCR and analyses were performed with a ViiA7 Real-time PCR System (Applied Biosystems; Thermo Fisher Scientific, Inc.). The $C$. elegans miRNA $39-3$ p was used as a normalization control. The specific primers for miRNA were purchased from Guangzhou RiboBio Co., Ltd. and their catalogue numbers were as follows: miR-324-5p forward, cat. no. ssD809230994; miR-630 forward, cat. no. ssD090525005; miR-324-5p and miR-630 reverse, cat. no. ssD089261711. Samples for target and reference gene amplification were prepared in triplicate. The gene expression levels were calculated using the $\Delta \mathrm{Ct}$ method (20). Divergent primers for circRNA were designed to amplify the circRNA-specific back-splice junctions and had the following sequences: hsa_circRNA_100906 forward, 5'-CTGGACAAGGCCACATAGAGT-3' and reverse, 5'-CAG AGCAGCCAATGAAGACAC-3'; hsa_circRNA_102100 forward, 5'-TCT ATTAGG GCATGAGTT TGT CTT-3' and reverse, 5'-TCCTTGGTTGTGGAGCTGTC-3'; hsa circRNA_102348 forward, 5'-CCTTTCAGCCCTCCATAC TTACT-3' and reverse, 5'-CCATATTCTTATCAGGCAATC TTGT-3'; hsa_circRNA_101225 forward, 5'-GCACCTGAC AGCATCTATTACC-3' and reverse, 5'-GACAGTAGAAAC GCAGTAAGCAA-3'; hsa_circRNA_104780 forward, 5'-ACA GATACCACCGCCGAACT-3' and reverse, 5'-TCTAGCTCC TTGGCAGGGAT-3'; hsa_circRNA_101242 forward, 5'-GAT GCTGCTCAAATGAGAAATG-3' and reverse, 5'-GCAGGA GAAGTATGTGGAGTAATC-3'. GAPDH forward, 5'-GGG AAACTGTGGCGTGAT-3' and reverse, 5'-GAGTGGGTG TCGCTGTTGA-3.'

Detection of putative miRNA seed matches. The miRNA response elements on circRNA and mRNA were scanned using miRNA target prediction software (version 1.0; Arraystar Inc.) based on miRanda and Target Scan. The differentially expressed circRNA within all comparisons was annotated in detail using the circRNA/miRNA interaction information. mRNA targets were obtained according to the target scores, which were calculated using the formula (ITargetScan context + scorel+|probabilities of conserved targeting|)/2.

Dual-luciferase reporter assay. Luciferase reporter assays were used to detect direct binding between circRNA and miRNAs. pMIR-REPORT Luciferase vector containing firefly luciferase gene (Obio Technology (Shanghai) Corp.,
Ltd. (Shanghai, China)) and pRL-cytomegalovirus Renilla luciferase reporter vector (Promega Corp., Madison, WI, USA) were applied in this experiment. The full length of the respective circRNA was obtained by gene synthesis (21). The synthesized circRNA was verified and inserted (21) into the pMIR-REPORT Luciferase vector by Obio Technology.

The mutant sequence was amplified from the wild-type using PCR and mutations in the target site was developed using primers. The mutant sequence was validated by sequencing following plasmid construction. The primer sequences used were as follows: has_circRNA_100906-F1, 5'-ATAGGCCGG CATAGACGCGTTGTCTTCATTGGCTGCTCTG-3'; hsa circRNA_100906-R1,5'-AAGGAGCCGTCGTACGGTCAT CCTGGGCTGTCAGATTTG-3'; hsa_circRNA _100906F2,5'-TGACCGTACGACGGCTCCTTTACATCTTGCTGC AACTCATG-3'; hsa_circRNA_100906-R 2,5'-TTCTTACCG TCGTACAAATCACCCTTAAAGGCAGCCA CATG-3'; hsa_circRNA_100906-F3,5'-GATTTGTACGACGGTAAGAAA TTAGTGGAAGATGGAGTAATC-3'; hsa_circRNA_ 100906-R3,5'AAAGATCCTTTATTAAGCTTCTCTTTG CCACATCACTGGG-3'; hsa_circRNA_102348-F1, 5'-ATA GGCCGGCATAGACGCGTTTTCAGAAAGTGCTTTCT CTC-3'; hsa_circRNA_102348-R1, 5'-GTTAACCCGTCG TAGTTTCCA AAGGTGCAACGCTCCGTGG-3'; hsa circRNA_102348-F2 ,5'-GGAAACTACGACGGGTTA ACCCAAGAGAGTGGACTCCAGAGA-3'; and hsa_ circRNA_102348-R2, 5'-AAAGATCCTTTATTAAGCTTA GTTTTTGAACTTCAGGCCACAACCGTCGTAGCAGAA GATGATCCT-3'. Mutations were introduced to verify the predicted miRNA binding sites. miRNA mimics and their negative control were obtained from Gene Pharma (Shanghai, China); they were co-transfected into 293T cells (Cell Bank of the Chinese Academy of Sciences, Shanghai, China) with the pMIR-REPORT Luciferase vector with or without the full-length sequence of circRNA by using Lipofectamine-2000 ${ }^{\circledR}$ (Invitrogen; Thermo Fisher Scientific, Inc.). After transfection for $48 \mathrm{~h}$, luciferase activities were detected with the dual-Luciferase Reporter Assay System (Promega Corp.) according to the manufacturer's protocols. Relative light units were determined with a SpectramaxM2 (Molecular Devices, LLC, Sunnyvale, CA, USA). Firefly luciferase values were normalized to the corresponding Renilla luciferase values.

Statistical analysis. Values are expressed as the mean \pm standard deviation. Student's t-test was used for comparison between two groups. Statistically significant differences from multiple groups were determined using one-way analysis of variance with the Student-Newman-Keuls post hoc test. All analyses were performed using SPSS Statistics software package (version 11.0 for Windows; SPSS, Inc., Chicago, IL, USA). $\mathrm{P}<0.05$ was considered to indicate a statistically significant difference.

\section{Results}

Identification of differentially expressed circRNA profiles during IPF development. IPF is clinically characterized by decreased lung function, increased high-resolution computed tomography evidence of honeycombing (but not emphysema), as well as significant microscopic honeycombing and 
A

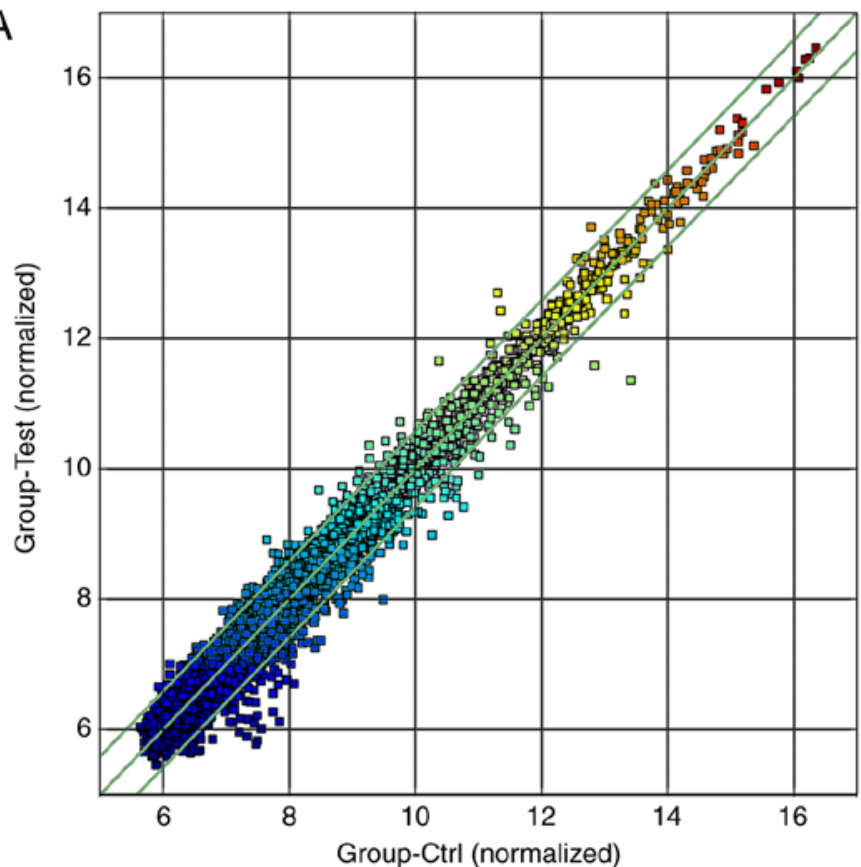

B
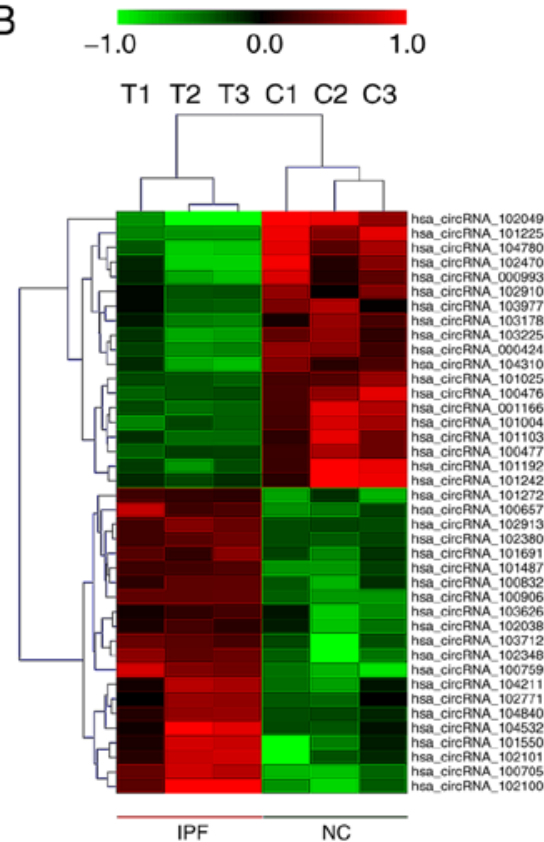

C
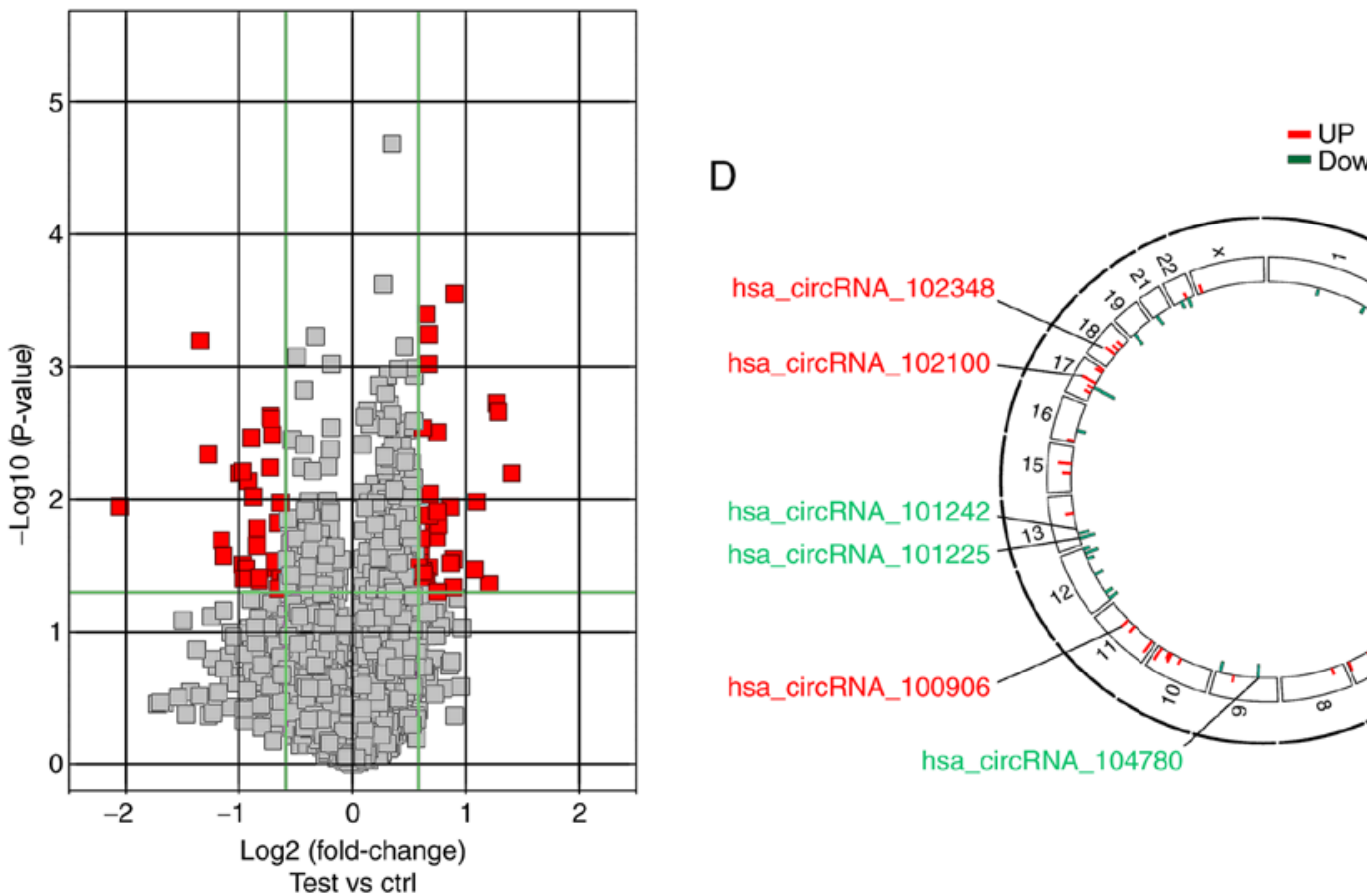

$\mathrm{D}$ - Down

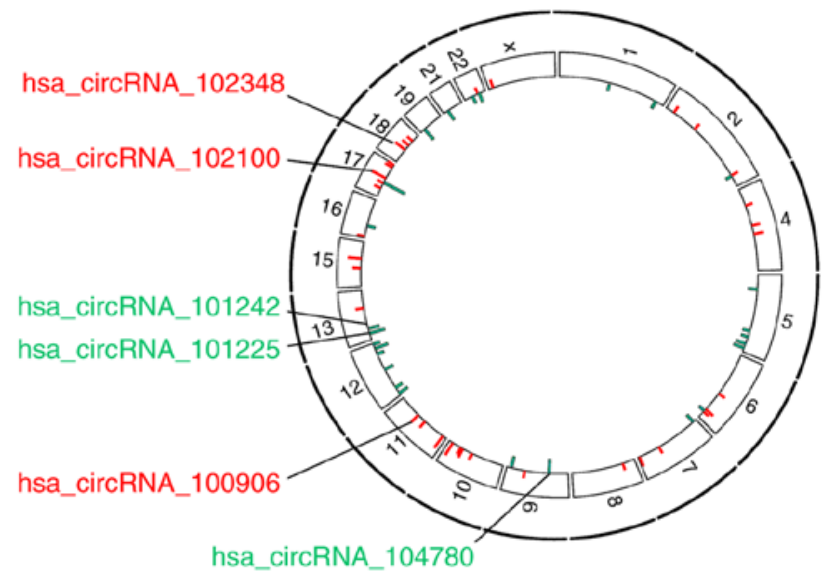

Figure 1. circRNA expression profile in IPF patients. (A) Variations (or reproducibility) of circRNA expression between the experiment and control groups

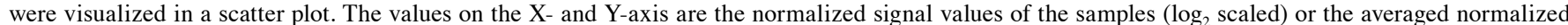
signal values of groups of samples $\left(\log _{2}\right.$ scaled). The green lines represent the fold change of 1.5. The circRNAs above the top green line and below the bottom green line indicate circRNAs with a fold change of $>1.5$ between the two compared samples. (B) Hierarchical clustering reveals the distinguishable circRNA expression profiling between IPF patients and normal human samples. Each column represents a sample and each row represents a circRNA. High or low relative expression is displayed as a red or green strip, respectively. Each group contains three different samples. (C) Volcano plots are useful tools for visualizing differential expression between two different conditions. The vertical lines correspond to a 1.5 -fold up- and downregulation, and the horizontal line represents a P-value of 0.05 . The red dots in the plot represent the differentially expressed circRNA with statistical significance. (D) Distribution of differentially expressed circRNAs in human chromosomes. Ctrl, control; IPF, idiopathic pulmonary fibrosis; circRNA, circular RNA; NC, normal control; hsa, Homo sapiens.

fibroblastic foci on pathology. The demographic and baseline characteristics of IPF patients and normal controls are presented in Table I. No significant differences in the patient number, age and gender between the normal and IPF groups were present.
A high-throughput microarray assay was used to identify circRNAs with abnormal expression in IPF. A total of 4,731 circRNA targets were detected using microarray probes in plasma samples from IPF patients and healthy individuals. The scatter plot in Fig. 1A displays the variation in the 

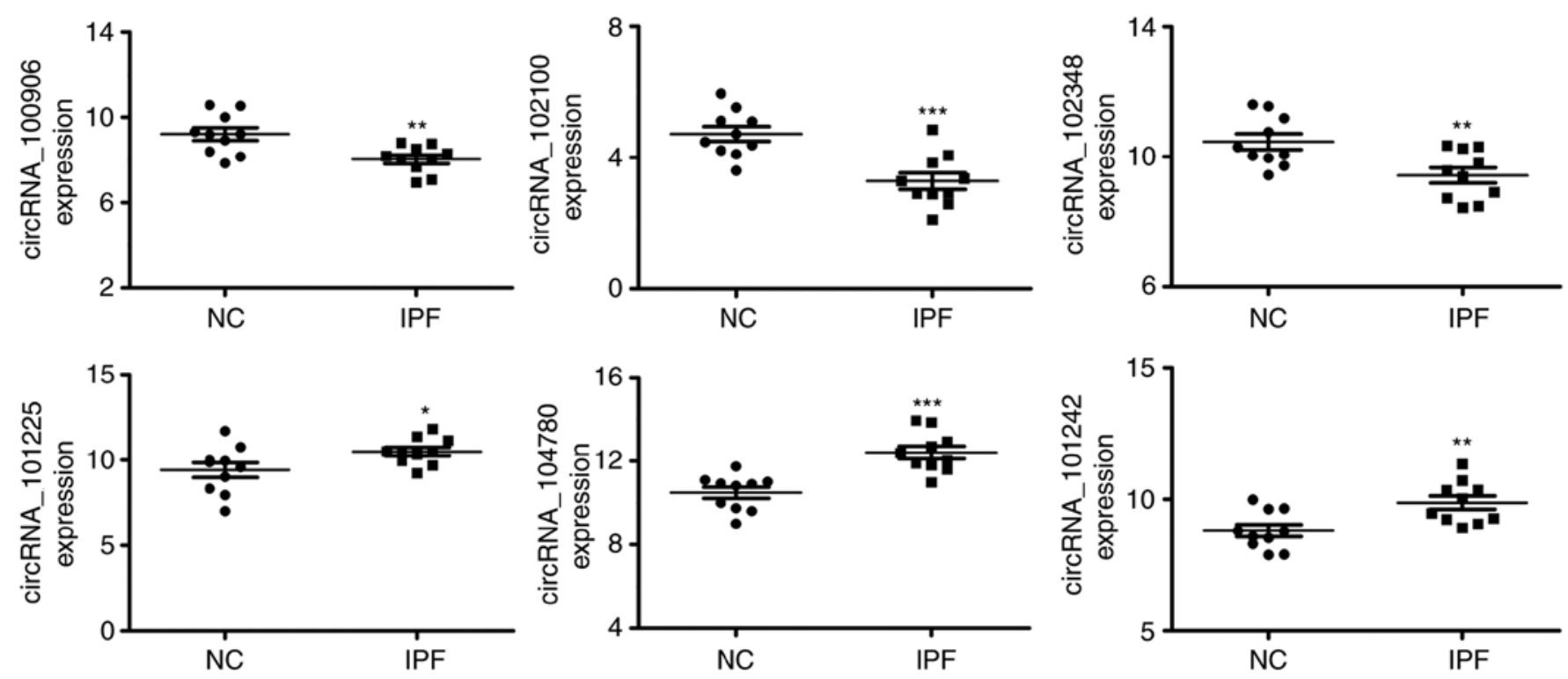

Figure 2. Validation of the differential expression of circRNAs in IPF and normal control samples. The expression levels of circRNAs were detected usingreverse-transcription quantitative polymerase chain reaction. Gene expression was quantified using the $\Delta \mathrm{Ct}$ method with normalization to GAPDH expression levels. An higher $\Delta \mathrm{Ct}$ value indicates lower expression. Values are expressed as the mean \pm standard deviation $(\mathrm{n}=10)$. ${ }^{*} \mathrm{P}<0.05$, ${ }^{* *} \mathrm{P}<0.01$ or ${ }^{* * * *} \mathrm{P}<0.001 \mathrm{vs}$. the NC determined via unpaired Student's t-tests. IPF, idiopathic pulmonary fibrosis; circRNA, circular RNA; NC, normal control.

Table I. Baseline characteristics and physiology of IPF patients and healthy individuals.

\begin{tabular}{lcc}
\hline Characteristic & $\begin{array}{c}\text { Controls } \\
(\mathrm{n}=10)\end{array}$ & $\begin{array}{c}\text { IPF patients } \\
(\mathrm{n}=10)\end{array}$ \\
\hline Age (years) & $67.5 \pm 8.7$ & $67.2 \pm 9.6$ \\
Gender (male/female) & $6 / 4$ & $6 / 4$ \\
FVC $(\%$ of predicted) & $88.9 \pm 10.2$ & $59.6 \pm 9.2^{\mathrm{a}}$ \\
FEV1/FVC $(\%$ of predicted) & $87.6 \pm 4.3$ & $85.5 \pm 2.4$ \\
TLC $(\%$ of predicted) & $88.1 \pm 13.2$ & $62.5 \pm 10.7^{\mathrm{a}}$ \\
$\mathrm{DLCO}(\%$ of predicted $)$ & $87.8 \pm 4.6$ & $53.3 \pm 10.6^{\mathrm{a}}$ \\
$\mathrm{PaO}_{2}(\mathrm{mmHg})$ & $86.1 \pm 2.7$ & $66.3 \pm 6.4^{\mathrm{a}}$ \\
$\mathrm{PaCO}_{2}(\mathrm{mmHg})$ & $39.7 \pm 3.4$ & $35.6 \pm 2.7$ \\
$\mathrm{Smoking} \mathrm{history}(\%)$ & 0 & 0 \\
\hline
\end{tabular}

${ }^{\text {ap }}<0.01$ vs. the normal group determined via unpaired Student's $\mathrm{t}$-tests. Values are expressed as the mean \pm standard deviation. Smoking history denotes subjects with $>5$ pack-years of cigarette smoking. FVC, forced vital capacity; FEV1/FVC, ratio of forced expiratory volume in the first second to forced vital capacity; TLC, total lung capacity; DLCO, diffusing capacity for carbon monoxide; IPF, idiopathic pulmonary fibrosis.

circRNA expression ratio between the two groups. The heat map of circRNA expression in samples ofthe two groups in Fig. 1B displays the regulation of several circRNA clusters. An upregulated cluster consisting of 38 circRNAs and a downregulated cluster consisting of 29 circRNAs were detected in the patients (fold change, $\geq 1.5 ; \mathrm{P}<0.05$ ). From the volcano plot in Fig. 1C, differentially expressed circRNAs with statistical significance between IPF and control samples were identified. The distribution of circRNA in human chromosomes is illustrated in Fig. 1D. A total of 21 and 18 of the most highly upregulated and downregulated circRNAs, respectively, according to their fold change, are listed in Table II.

Subsequently, six differentially expressed circRNA (hsa_circRNA_100906, hsa_circRNA_102100, hsa circRNA_102348,hsa_circRNA_101225,hsa_circRNA_104780 and hsa_circRNA_101242) were selected for further validation and analysis due to their high fold-changes and low P-values. The expression levels were quantified via RT-qPCR with circRNA-specific divergent primers calibrated using standard curves with housekeeping genes as normalization standards. The RT-qPCR results validated the differential expression of these circRNAs by using 10 independent samples. The results indicated that, hsa_circRNA_100906, hsa_circRNA_102100 and hsa_circRNA_102348 were significantly upregulated, whereas hsa_circRNA_101225, hsa_circRNA_104780 and hsa_circRNA_101242 were significantly downregulated in the IPF vs. normal control samples (Fig. 2). The changes in the expression of certain circRNAs identified from the overall array data were therefore in accordance with the results of theRT-qPCR measurements.

Genomic location of differentially expressed circRNAs in $I P F$ disease. circRNA is typically generated at the expense of canonical mRNA isoforms, thereby indicating its possible function as an important regulator of mRNA production (22). To predict the function of the circRNAs, their location in the human genome was first detected. Differentially expressed circRNA was typically derived from annotated exons (86.6\%). Its splice sites typically span 1-11 exons and overlap the coding exon. Only small fractions of circRNA originated from introns or were aligned antisense regionsto known transcripts (Fig. 3A).

Several differentially expressed circRNAs originated from known protein-coding genes with pivotal roles in fibrosis [e.g. zinc-finger-DHHC-type with 4 (ZDHHC4)]. Kyoto 
Table II. Most differentially expressed circRNA ranked by FC vs. NC group in microarray data.

\begin{tabular}{|c|c|c|c|c|c|c|}
\hline circRNA & P-value & $\mathrm{FC}(\mathrm{abs})$ & Change vs. NC & Alias & circRNA_type & Gene symbol \\
\hline hsa_circRNA_100906 & 0.00028136 & 1.8654768 & Upregulation & hsa_circ_0023858 & Exonic & ANKRD42 \\
\hline hsa_circRNA_102380 & 0.000400941 & 1.5656011 & Upregulation & hsa_circ_0047841 & Exonic & LIG3 \\
\hline hsa_circRNA_102913 & 0.000949382 & 1.5911579 & Upregulation & hsa_circ_0058058 & Exonic & ATIC \\
\hline hsa_circRNA_100705 & 0.001874312 & 2.4096969 & Upregulation & hsa_circ_0008898 & Exonic & OAT \\
\hline hsa_circRNA_100759 & 0.002180791 & 2.4327399 & Upregulation & hsa_circ_0004099 & Exonic & DENND5A \\
\hline hsa_circRNA_102100 & 0.006302884 & 2.6442716 & Upregulation & hsa_circ_0044226 & Exonic & $\mathrm{CDC} 27$ \\
\hline hsa_circRNA_102348 & 0.010380106 & 2.1353111 & Upregulation & hsa_circ_0007535 & Exonic & ELP2 \\
\hline hsa_circRNA_102101 & 0.033608302 & 2.1090716 & Upregulation & hsa_circ_0044234 & Exonic & $\mathrm{CDC} 27$ \\
\hline hsa_circRNA_101550 & 0.043316607 & 2.3059601 & Upregulation & hsa_circ_0035796 & Exonic & HERC1 \\
\hline hsa_circRNA_100657 & 0.011363912 & 1.8185249 & Upregulation & hsa_circ_0006520 & Exonic & MMS19 \\
\hline hsa_circRNA_103712 & 0.02802823 & 1.8542931 & Upregulation & hsa_circ_0007540 & Exonic & TBCK \\
\hline hsa_circRNA_104211 & 0.030050202 & 1.8239174 & Upregulation & hsa_circ_0007762 & Exonic & STXBP5 \\
\hline hsa_circRNA_104532 & 0.04568836 & 1.8495971 & Upregulation & hsa_circ_0001772 & Exonic & RBM33 \\
\hline hsa_circRNA_102038 & 0.034423041 & 1.541653 & Upregulation & hsa_circ_0043082 & Exonic & LIG3 \\
\hline hsa_circRNA_104840 & 0.013111444 & 1.5986307 & Upregulation & hsa_circ_0002780 & Exonic & CDC14B \\
\hline hsa_circRNA_101487 & 0.003102427 & 1.6784173 & Upregulation & hsa_circ_0008926 & Exonic & NUSAP1 \\
\hline hsa_circRNA_103626 & 0.040907511 & 1.5692823 & Upregulation & hsa_circ_0007308 & Exonic & PDS5A \\
\hline hsa_circRNA_102771 & 0.049588502 & 1.5610834 & Upregulation & hsa_circ_0055377 & Exonic & CTNNA2 \\
\hline hsa_circRNA_101691 & 0.009068437 & 1.6051068 & Upregulation & hsa_circ_0007637 & Exonic & CREBBP \\
\hline hsa_circRNA_100832 & 0.014303719 & 1.6414589 & Upregulation & hsa_circ_0022378 & Exonic & FADS1 \\
\hline hsa_circRNA_101272 & 0.012972773 & 1.6474556 & Upregulation & hsa_circ_0005783 & Exonic & KLF12 \\
\hline hsa_circRNA_101225 & 0.000635675 & 2.5487789 & Downregulation & hsa_circ_0029633 & Exonic & ZMYM2 \\
\hline hsa_circRNA_101025 & 0.002349167 & 1.6468162 & Downregulation & hsa_circ_0025633 & Exo & LRMP \\
\hline hsa_circRNA_104780 & 0.00453198 & 2.4221486 & Downregulation & hsa_circ_0001861 & Exonic & GRHPR \\
\hline hsa_circRNA_101004 & 0.006100264 & 1.9569623 & Downregulation & hsa_circ_0000375 & Exonic & IFFO1 \\
\hline hsa_circRNA_102049 & 0.011284484 & 4.1574829 & Downregulation & hsa_circ_0043278 & Exonic & TADA2A \\
\hline hsa_circRNA_101192 & 0.020134914 & 2.2308587 & Downregulation & hsa_circ_0005465 & Exonic & CLIP1 \\
\hline hsa_circRNA_102470 & 0.0264833 & 2.2000704 & Downregulation & hsa_circ_0049888 & Exonic & EPS15L1 \\
\hline hsa_circRNA_000993 & 0.030740567 & 1.9544226 & Downregulation & hsa_circ_0001887 & Intragenic & STRBP \\
\hline hsa_circRNA_101242 & 0.03320098 & 1.9118807 & Downregulation & hsa_circ_0029853 & Exonic & PAN3 \\
\hline hsa_circRNA_100477 & 0.005705005 & 1.6533506 & Downregulation & hsa_circ_0016867 & Exonic & COG2 \\
\hline hsa_circRNA_100476 & 0.007246795 & 1.8857807 & Downregulation & hsa_circ_0016863 & Exonic & COG2 \\
\hline hsa_circRNA_001166 & 0.006309977 & 1.9935227 & Downregulation & hsa_circ_0001556 & Intronic & ERGIC1 \\
\hline hsa_circRNA_103225 & 0.009486195 & 1.8307713 & Downregulation & hsa_circ_0063331 & Exonic & DDX17 \\
\hline hsa_circRNA_104310 & 0.016308893 & 1.7897346 & Downregulation & hsa_circ_0079385 & Exonic & ZDHHC4 \\
\hline hsa_circRNA_000424 & 0.003435538 & 1.8528501 & Downregulation & hsa_circ_0001549 & Antisense & FABP6 \\
\hline hsa_circRNA_103977 & 0.046667432 & 1.5710154 & Downregulation & hsa_circ_0074362 & Exonic & ARHGAP26 \\
\hline hsa_circRNA_103178 & 0.029174857 & 1.6035583 & Downregulation & hsa_circ_0062577 & Exonic & CABIN1 \\
\hline hsa_circRNA_102910 & 0.041706263 & 1.5010713 & Downregulation & hsa_circ_0058051 & Exonic & BARD1 \\
\hline
\end{tabular}

Regarding the circRNA type, circRNA is classified into four categories: Exonic, intronic, antisense and intergenic. The P-value was calculated using anunpaired Student's t-test. FC (abs), absolutefold change; Alias, circRNA ID in the circ Base database (circbase.mdc-berlin.de); circRNA, circular RNA; NC, normal control; hsa, Homo sapiens.

Encyclopaedia of Genes and Genomes (KEGG) analysis revealed that genes that produced dysregulated circRNAs were involved in the pathways of cell cycle, adherens junctions, ubiquitin-mediated proteolysis, and RNA transport and degradation (Fig. 3B and C).

As presented in Fig. 3D-I, the tested circRNAs all belonged to exons. In addition, exonic circRNAs may result from spli- ceosomal action, e.g. the exon structure of the human ankyrin repeat domain 42 locus, encompassing a 797-nt region that includes exons 3-8.

Predicted ceRNA network of circRNAs/miRNAs/mRNAs in $I P F$. A considerable number of studies have reported that circRNAs act as 'miRNA sponges' to regulate gene expres- 

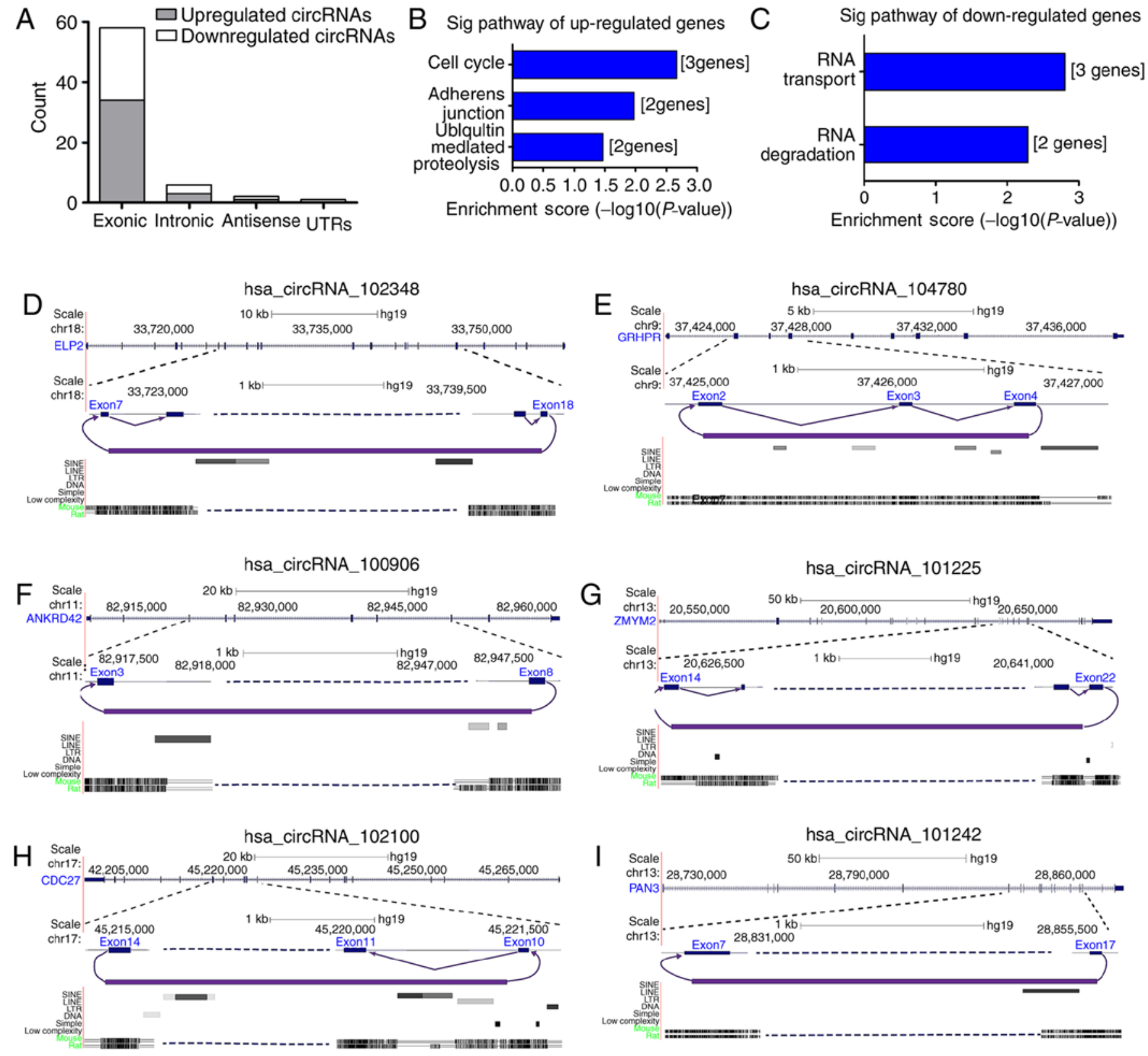

Figure 3. Correlation of differential expression of circRNAs with their host genes. (A) Bar graph presenting the circRNA category. (B and C) Results of a Kyoto Encyclopedia of Genes and Genomes pathway analysis displaying the participant pathways of host genes. (D-I) Designated orientations and exon structure of detected six circRNA. For example, has_circRNA_104780 is formed when the 5' splice site at the end of exon 4 is joined to the 3' splice site at the beginning of exon 2 (purple). circRNA, circular RNA; Chr, chromosome; Sig, significantly enriched, UTR, untranslated region; hsa, Homo sapiens. ELP2, elongator acetyltransferase complex subunit 2; GRHPR, glyoxylate and hydroxypyruvate reductase; ANKRD42, ankyrin repeat domain 42; ZMYM2, zinc finger MYM-type containing 2; CDC27, cell division cycle 27; PAN3, poly(A) specific ribonuclease subunit PAN3.

sion. The present study evaluated the biological role of differentially expressed circRNAs by drawing and analysing an miRNA-mediated regulatory network. The number of predicted miRNA binding sites for all miRNAs (deposited in miR Base version 19) was determined. Interactions between circRNAs and miRNAs were theoretically predicted according to their conserved seed-matching sequences. The analysis revealed that all 67 of the differentially expressed circRNAs contained response elements for their respective target miRNAs. The top five miRNAs for each 67 circRNAs were displayed as a network delineated using Cytoscape software (version 3.1.0; cytoscape.org/) (Fig. 4A). A sub-network displaying 10 circRNAs and their target miRNAs is displayed in Fig. 4B. Numerous associated miRNAs have pivotal roles in the progression of fibrotic disease (including miR-326, -361-5p, -338-3p, -9-5p, -136-5p, -145-5p, -224-5p and $-877-3 p)$. On the basis of the number of binding sites and target mRNAs, miR-330-5p/miR-324-5p, miR-532-5p, miR-630, miR-326, miR-650 and miR-338-3p/miR-21-3p were selected as potential miRNA targets for the six selected circRNAs (hsa_circRNA_100906, hsa_circRNA_102100, hsa_circRNA_102348, hsa_circRNA_101225, hsa_ circRNA_104780 and hsa_circRNA_101242, respectively). The sequence analysis of the miRNA response elements for the six selected differentially expressed circRNAs and their potential complementary binding miRNAs is displayed in Fig. 5. 
A

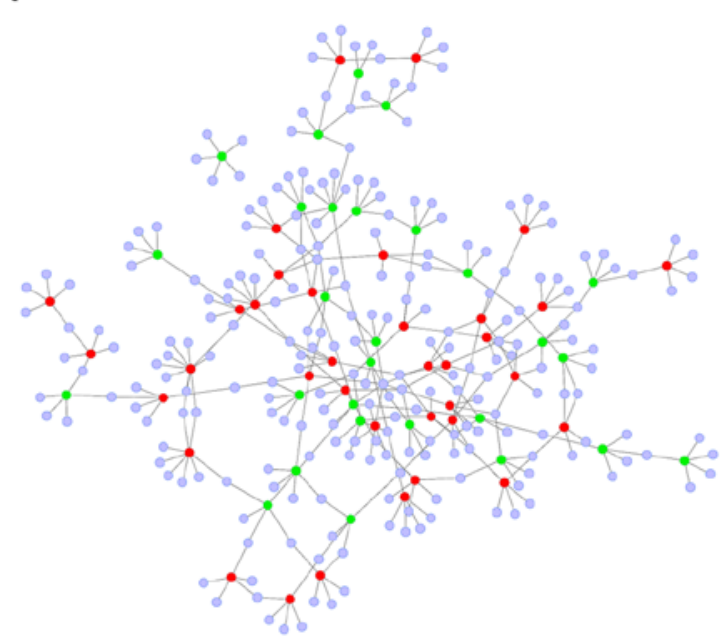

B

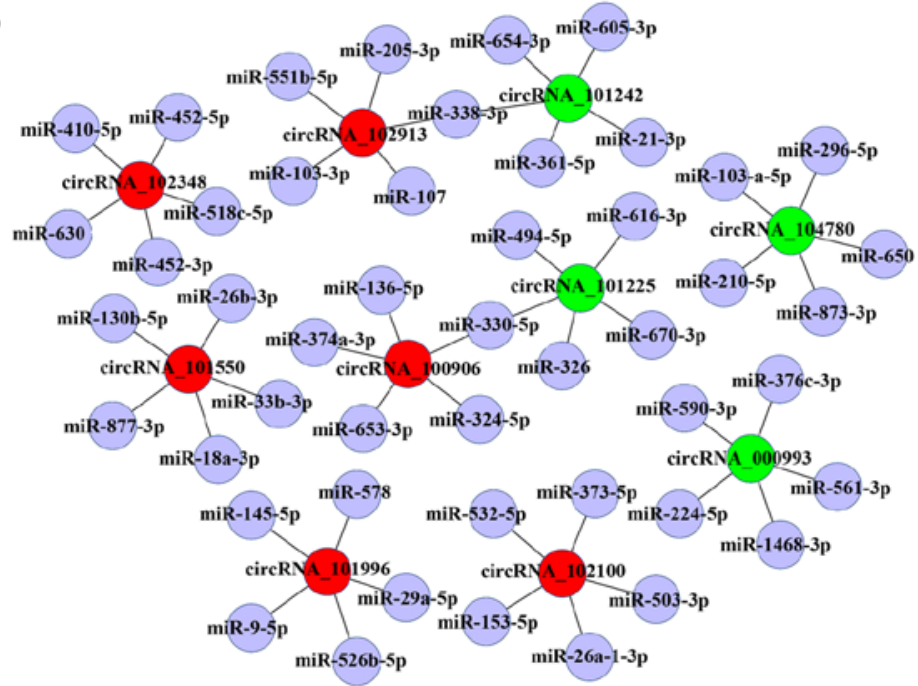

Figure 4. Interaction network of circRNAs and miRNAs. (A) circRNA-miRNA interaction network consisting of 38 upregulated circRNAs, 29 downregulated circRNAs and their target miRNAs. They were connected by 333 edges based on seed sequence pairing interactions. (B) Interaction of 10 circRNAs (including 6 verified circRNAs: hsa_circRNA_100906, hsa_circRNA_102100, hsa_circRNA_102348, hsa_circRNA_101225, hsa_circRNA_104780 and hsa_circRNA_101242; and 4 circRNAs with high expression and predicted binding, which serves an important role in lung fibrosis) and their target miRNAs presented in a magnified network. Red and green nodes represent upregulated and downregulated circRNAs, respectively. Purple nodes indicate miRNAs. miR/miRNA, microRNA; circRNA, circular RNA; hsa, Homo sapiens.

\section{A}

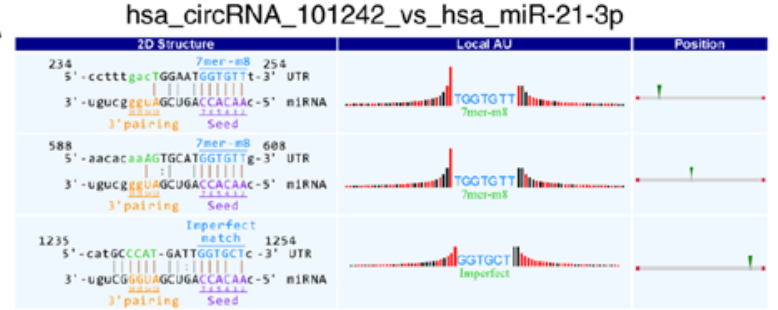

C

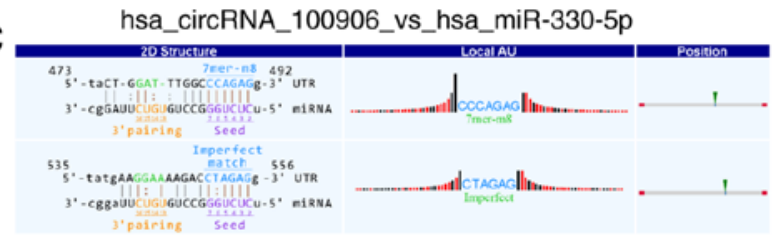

$\mathrm{E}$

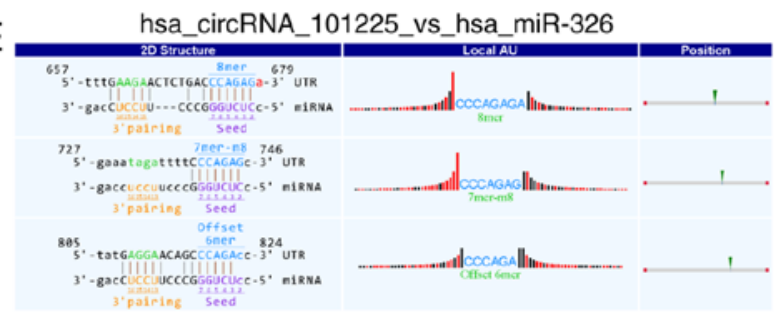

$\mathrm{G}$

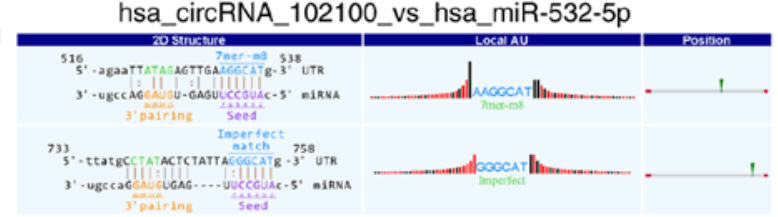

$\mathrm{B}$

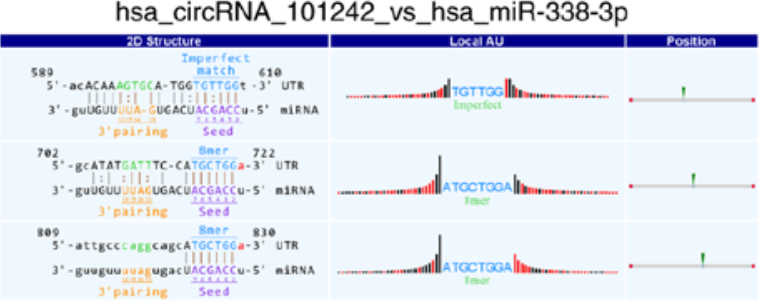

$\mathrm{D}$

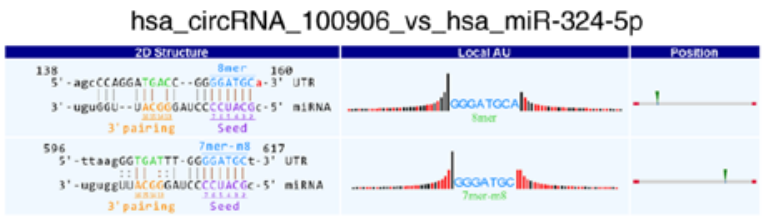

$\mathrm{F}$

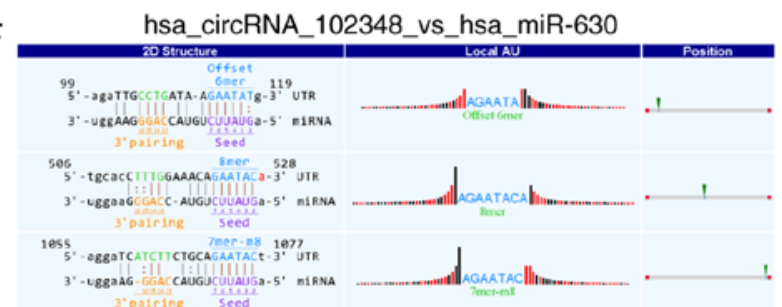

$\mathrm{H}$

hsa_circRNA_104780_vs_hsa_miR-650

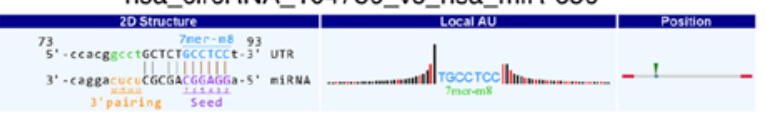

Figure 5. Detailed annotation for circRNA/miRNA interaction based on TargetScan and miRanda. The '2D Structure' column presents the sequences of circRNA and miRNA. The 'Local AU' column displays 30 nucleotides upstream and downstream of the seed sequence. The 'Position' column indicates the probable position of the miRNA response element on the circRNA. (A and B) Interaction between hsa_circRNA_101242 and hsa_miR-21-3p or hsa_ miR-338-3p, respectively. (C and D) Interaction between hsa_circRNA_100906 and hsa_miR-330-5p or hsa_miR-324-5p, respectively. (E-H) Display of the interaction of (E) hsa_circRNA_101225 and hsa_miR-326, (F) hsa_circRNA_102348 and hsa_miR-630, (G) hsa_circRNA_102100 and hsa_miR-532-5p and (H) hsa_circRNA_104780 and hsa_miR-650. miR/miRNA, microRNA; circRNA, circular RNA; UTR, untranslated region; hsa, Homo sapiens. 

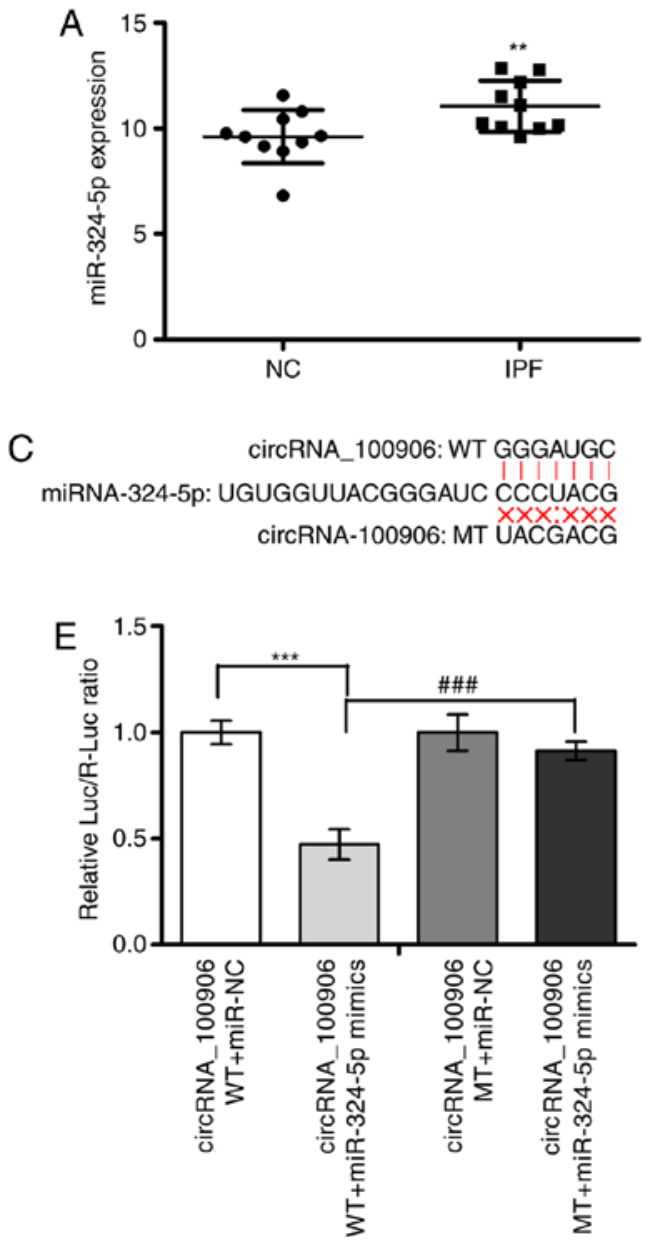
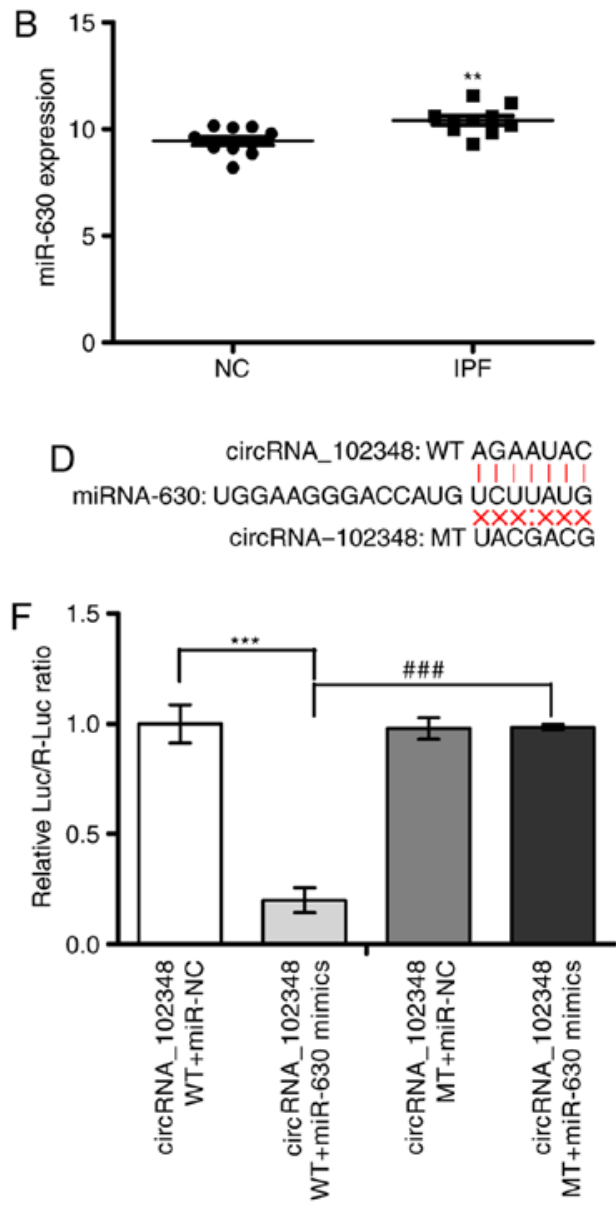

Figure 6. Direct interaction between circRNA and miRNA. (A and B) Expression levels of miR-630 and miR-324-5p in IPF patients. $\Delta$ Ct values were determined to quantify gene expression. Higher $\Delta \mathrm{Ct}$ value indicates lower expression. C. elegans miRNA $39-3 p$ was used as a normalization control. Values are expressed as the mean \pm standard deviation $(\mathrm{n}=10)$. ${ }^{* *} \mathrm{P}<0.01$ vs. the NC group measured via unpaired Student's t-tests. (C) Wild-type and mutated miR-324-5p binding site on hsa_circRNA_100906. (D) hsa_circRNA_102348 sequences containing wild-type or mutated miR-630 binding sites. (E and F) Dual-luciferase reporter assay demonstrated direct binding or miR-324-5p and miR-630 with circRNA-100906 and circRNA-102348, respectively. Values are expressed as the mean \pm standard deviation. ${ }^{* * *} \mathrm{P}<0.001$; ${ }^{\# \# \#} \mathrm{P}<0.001$ measured via analysis of variance and Student-Newman-Keuls test. miR/miRNA, microRNA; IPF, idiopathic pulmonary fibrosis; circRNA, circular RNA; NC, normal control; WT, wild-type; MT, mutated type; Luc, luciferase; R, Renilla.

For instance, it was revealed that in hsa_circRNA_100906, the 154 to 159 th and the 611 to 616th nucleotides starting from the 5 ' terminus were completely complementary to the miR-324-5p seed region in the 8 mer or 7 mer-m8 binding mode.

Subsequently, circRNA_100906/miR-324-5p and circRNA_102348/miR-630 were selected to confirm the predicted interaction between circRNA and miRNA by luciferase reporter assays. The expression levels of miR-324-5p and miR-630 were significantly downregulated in IPF patients conversely to those of their associated circRNAs (Fig. 6A and B). The luciferase intensity decreased by $>50 \%$ with the co-transfection of luciferase reporters containing the circRNA_100906 or circRNA_102348 full length sequence and mimics of miR-324-5p or miR-630, respectively. To confirm the direct interaction, the miRNA response elements (MREs) in the luciferase reporter were mutated. Co-transfection of miRNA mimics and luciferase reporter vector with the mutated sequence did not considerably affect the luciferase activity (Fig. 6C-F).

miR-330-5p/miR-324-5p, miR-532-5p, miR-630, miR-326, miR-650 and miR-338-3p/miR-21-3p were predicted as target genes and theregulatory network of circRNAs/miRNAs/mRNAs is presented in Fig. 7A. Numerous fibrosis-associated genes [e.g. SMAD3, ECM protein 1 (ECM1), vimentin, suppressor of cytokine signalling 1 (SOCS1), rho-associated kinase (ROCK) 1, matrix metalloproteinase (MMP1), integrin subunit $\beta$ like 1 (ITGBL1), ADAM metallopeptidase domain 17 and hypoxia-inducible factor 1 subunit $\alpha$ (HIF1A) inhibitor] may be targeted by the previously mentioned miRNAs. The circRNA/miRNA regulatory networks act on target genes significantly involved in transforming growth factor (TGF)- $\beta 1$, HIF-1, Wnt, Janus kinase (JAK), ROCK, vascular endothelial growth factor, mitogen-activated protein kinase, Hedgehog and nuclear factor $\kappa \mathrm{B}$ signalling pathways, which are associated with cell proliferation, migration and collagen synthesis (Fig. 7B). For instance, circRNA_100906 may trigger the upregulation of SOCS1, ROCK1, SP1, nemo-like kinase, MMP1, bromodomain containing 2, ECM1, ITGBL1, phosphoinositide-3-kinase regulatory subunit 3 and fibroblast growth factor receptor 2 by sequestering miR-324-5p and miR-330-5p in the network. The ceRNA network and associated pathway components may be novel clinical markers and therapeutic targets for IPF. 


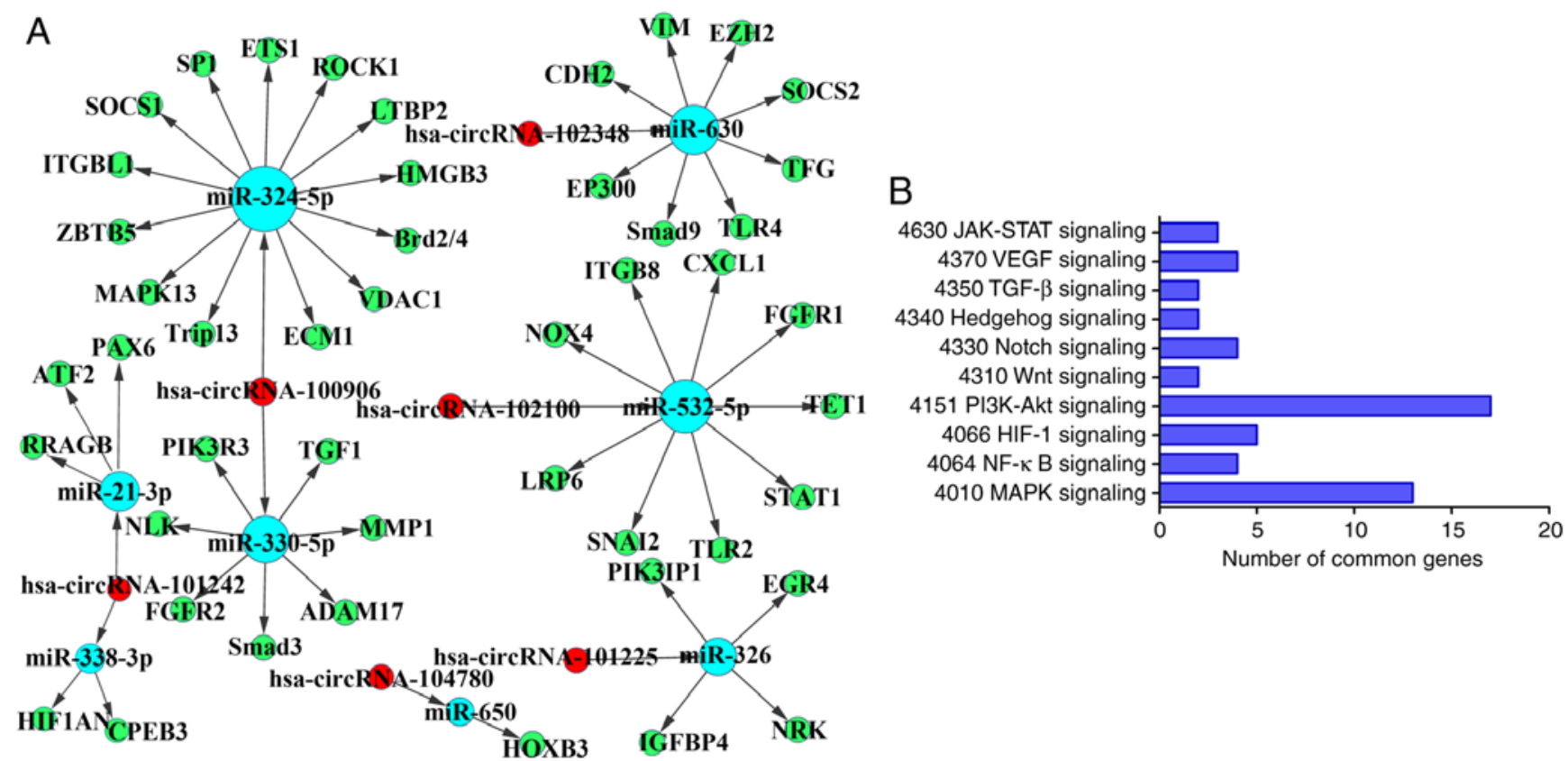

Figure 7. Prediction ofcompeting endogenous RNA network of circRNAs/miRNAs/mRNAs. (A) Bioinformatics prediction of the circRNA/miRNA/mRNA network. Red represents circRNA, green represents mRNA and blue represents miRNA. (B) Kyoto Encyclopedia of Genes and Genomes analysis of pathways enriched by the targeted mRNAs. miR/miRNA, microRNA; circRNA, circular RNA. JAK, Janus kinase; STAT, signal transducer and activator of transcription; PI3K, phosphoinositide-3 kinase; HIF, hypoxia-inducible factor; TGF, transforming growth factor; VEGF, vascular endothelial growth factor; MAPK, mitogen-activated protein kinase.

\section{Discussion}

circRNAs are regarded as novel clinical diagnostic, prognostic and therapeutic biomarkers that may provide novel approaches for the treatment of diseases (23). For instance, the expression of circular ANRIL is correlated with the risk for atherosclerotic vascular disease (24). However, whether circRNAs have a role in IPF has remained elusive. In the present study, circRNA expression profiling was performed in IPF patients and 67 aberrantly expressed circRNAs were identified. Furthermore, it was unveiled that certain circRNAs (hsa_circRNA_100906, hsa_circRNA_102100 and hsa_circRNA_102348) were upregulated, while others (hsa_circRNA_101225, hsa_circRNA_104780 and hsa_ circRNA_101242) were downregulated in IPF.

circRNA originates from exons, introns or is aligned with antisense regions to known transcripts, intergenic sequences or unannotated regions of the genome (25). Linear splicing and circRNA production compete against each other for splicing sites and assign a regulated function to circRNA in their hosted gene (26). Chao et al (27) reported that circRNA originating from the mouse formin (Fmn) gene functioned as an 'mRNA trap' by leaving a non-coding linear transcript, thereby reducing the expression levels of the Fmn protein. circRNA to eukaryotic translation initiation factor 3 subunit $\mathrm{J}$ and poly(A) binding protein interacting protein 2 are predominantly localized in the nucleus, interacting with U1 small nuclear ribonucleoproteins and enhancing the transcription of their parental genes in a cis-acting manner (28). In the present study, numerous parental genes of differentially expressed circRNAs associated with the biological process of fibrosis were identified. Yang et al (29) reported that ZDHHC4, which causes a significant downregulation of circRNAhsa_circ_104310, is a significant methylation marker to regulate the expression of large groups of genes in a trans-acting manner in IPF. André et al (30) demonstrated that BRCA1-associated RING domain 1, the host gene of hsa_circRNA_102910, regulates lung epithelial cell damage and fibroblast proliferation by acting as a mediator of hypoxia and TGF- $\beta$ may be a novel target for IPF treatment. hsa_circRNA_102348 is spliced from the elongator acetyltransferase complex subunit 2 gene, which encodes a general binding partner or chaperone for the activation and nuclear translocation of signal transducer and activator of transcription (STAT)3 (31) and may regulate the JAK/STAT signalling pathway. hsa_circRNA_102100 and 102101 align with the gene cell division cycle (CDC)27, which encodes a core component of the anaphase-promoting complex/cyclosome. Furthermore, aberrant expression of CDC27 may result in chromosomal aneuploidy integrity and improper cell cycle progression, which may have roles in cell proliferation in IPF (32). The parental gene of hsa_circRNA_101225 encodes a zinc finger MYM type 2, which apparently binds specifically to fibroblast growth factor receptor-1 or proteins with added small ubiquitin-like modifiers, including histone deacetylase 1 (33). KEGG analysis supported the concept of target genes that may regulate crucial biological processes during the development of IPF.

The highly complex regulatory network of circRNA/miRNA/mRNA represents another important layer of epigenetic control over gene expression in health and disease, involving a variety of cellular processes, including the cell cycle, apoptosis and adherens junctions $(34,35)$. At present, sponging activity is the major function of certain circRNAs. A $1.2-\mathrm{kb}$ single-exon circRNA produced from 
the mammalian sex determination gene may function as a miR-138 sponge for 16 target sites (36). The circRNA/miRNA interaction analysis of the present study indicated that abnormally expressed circRNAs in IPF patients possess abundant miRNA target sites that have important roles in fibrotic disease. Zhang et al (37) reported that the miR-338-5p/lysophosphatidic acid receptor 1 axis may be regarded as a target of tectorigenin in bleomycin-induced lung fibrosis. In the present study, miR-338-5p was matched with hsa_circRNA_102101 and hsa_circRNA_102100, which originate from the same coding gene (CDC27). hsa_circRNA_101996 possessed MREs for miR-9 and -145 , which regulated fibrosis by targeting platelet-derived growth factor receptor $\beta /$ extracellular signal-regulated kinase signalling or the TGF- $\beta$ receptor 2/SMAD3/TGF- $\beta$ pathway $(38,39)$.

miRNAs including miR-324-5p, miR-330-5p, miR-532-5p, miR-630, miR-650, miR-21-3p and miR-326 reportedly participate in cell proliferation, apoptosis, migration, epithelial-mesenchymal transition (EMT), and cytoskeleton remodelling regarding as tumour markers $(40,41)$. However, limited information is available regarding their role in fibrotic disease. Pulmonary fibrosis is a neoproliferative disorder of the lung that exhibits several cancer-like pathogenic features, including abnormal activation, uncontrolled proliferation, resistance to apoptosis and high migration rates of myofibroblasts. These miRNAs were perfectly matched with the six circRNAs selected in the present study. MMP1, Snai2 and vimentin, which are the predicted target genes of miR-330-5p, 532-5p and 630, respectively, are mostly accepted as biomarkers for fibrosis. Early growth response 4, which contains binding sites for miR-326, reportedly counteracts autocrine TGF- $\beta$ signalling and abolishes myofibroblast function (42). As a co-receptor for Wnt, low-density lipoprotein receptor related protein 5 and -6 are predicted targets of miR-532-5p, transmitting the canonical Wnt/ $\beta$-catenin signalling and appearing independently associated with fibrotic disease progression (43). KEGG analysis further demonstrated the involvement of the target genes in fibrosis-associated signalling pathways.

circRNA_000203 is regarded as a ceRNA of collagen type I alpha 2 chain and connective tissue growth factor by interacting with miR-26b-5p in cardiac fibrosis (44). Furthermore, $\mathrm{Zhou}$ and $\mathrm{Yu}$ (45) reported that the profibrotic function of circRNA_010567 is partly mediated by the miR-141/TGF- $\beta 1$ pathway. However, differential expression of the two circRNAs and the associated miRNAs was not observed in the present study. circRNA_101242, which harbours two binding sites for miR-21-3p, has been reported to be abnormally expressed in gastric cancer (46). In the present study, circRNA_101242 was also verified to be downregulated, whereas miR-21-3p was significantly upregulated in IPF. The direct binding and target mRNA require verification in further studies. A previous study reported that circRNA_100269 prevents gastric cancer proliferation by sponging miR-630 (47). However, in the present study, no evident change of circRNA_100269 was noted, whereas miR-630 was significantly downregulated in IPF patients. In addition, circRNA_102348, which harbours two MREs for miR-630, was upregulated and demonstrated to directly interact with miR-630. Previous studies have only identified a small number of circRNAs or ceRNAs with multiple binding sites for a particular miRNA; most
circRNAs or ceRNAs were identified to contain only one or two miRNA binding sites (48). The predicted number of miRNA binding sites in differentially expressed circRNAs in the present study was three at the most as determined by Arraystar's prediction software. Furthermore, the interaction of circRNA_100906/miR-324-5p was identified in the present study. As a predicted target gene of miR-630, E1A binding protein p300was identified as a co-activator of HIF1A, which is responsible for lung fibrosis (49). Li et al (50) reported that the H19/miR-630/enhancer of zeste homolog 2(EZH2) signalling pathway has a considerable role in nasopharyngeal carcinoma metastasis. The activation of ROCK1 and -2 , which are targeted by miR-324-5p according to the present analysis, is involved in actin filament assembly, actomyosin contraction, cell adhesion and motility, proliferation and apoptosis, and the remodelling of the ECM in lung fibrosis (51). miR-324-5p suppresses ECM degradation in hepatocellular carcinoma by post-transcriptionally downregulating ETS proto-oncogene 1, transcription factor and SP1 (52). Furthermore, ITGBL1, the predicted target gene of miR-324-5p, wasidentified to be significantly overexpressed in the lung tissue of bleomycin-injured mice and TGF- $\beta 1$-treated cells, which inhibited EMT, myofibroblast migration and collagen synthesis (data not shown). The direct target genes of miR-630 and miR-324-5p in IPF will be further explored in future studies by our group.

In conclusion, the present study indicated that dysregulated circRNAs function as important regulators for pro- or anti-fibrotic signalling pathways in IPF by sequestering miRNAs or regulating their host genes. The important roles of circRNAs in IPF will be considered for further studies in our group. Ongoing efforts will be made to provide additional fundamental information for improving the understanding of the regulatory mechanisms in IPF and to provide novel approaches for the diagnosis, treatment and prevention of the disease.

\section{Acknowledgements}

Not applicable.

\section{Funding}

This study was supported by National Natural Science Foundation of China (81273957, 31300288, 31470415, 81670064, 31670365 and 81530030), Natural Science Foundation of Shandong Province (2014GSF119014 and ZR2016HP34) and The project of Traditional Medical Science and Technology for Shandong Province (2015-271).

\section{Availability of data and materials}

The analysed data sets generated during the study are available from the corresponding author on reasonable request.

\section{Authors' contributions}

RL, YW and XS performed the experiments, analysed the data and wrote the paper. WS, YL, JZ and QZ performed the experiments and analysed the data. HL and CM collected the 
clinical samples and analysed the data. JZ and CL conceived the experiment, analysed the data and wrote the paper.

\section{Ethics approval and consent to participate}

The present study was performed in accordance with the Declaration of Helsinki and the protocol was approved by the Ethics Committee of Binzhou Medical University (Binzhou, China). Written informed consent was obtained from the participants of the present study.

\section{Patient consent for publication}

Not applicable.

\section{Competing interests}

The authors declare that they have no competing interests.

\section{References}

1. Selman M and Pardo A: Stochastic age-related epigenetic drift in the pathogenesis of idiopathic pulmonary fibrosis. Am J Respir Crit Care Med 190: 1328-1330, 2014.

2. Clarke DL, Murray LA, Crestani B and Sleeman MA: Is personalised medicine the key to heterogeneity in idiopathic pulmonary fibrosis? Pharmacol Ther 169: 35-46, 2017.

3. Tzouvelekis A, Yu G, Lino Cardenas CL, Herazo-Maya JD, Wang R, Woolard T, Zhang Y, Sakamoto K, Lee H, Yi JS, et al $\mathrm{SH} 2$ domain-containing phosphatase-2 is a novel antifibrotic regulator in pulmonary fibrosis. Am J Respir Crit Care Med 195: 500-514, 2017.

4. Richeldi L, Collard HR and Jones MG: Idiopathic pulmonary fibrosis. Lancet 389: 1941-1952, 2017.

5. Mao C, Zhang J, Lin S, Jing L, Xiang J, Wang M, Wang B, Xu P, Liu W, Song X and Lv C: MiRNA-30a inhibits AECs-II apoptosis by blocking mitochondrial fission dependent on Drp-1. J Cell Mol Med 18: 2404-2416, 2014.

6. Cao G, Zhang J, Wang M, Song X, Liu W, Mao C and Lv C: Differential expression of long non-coding RNAs in bleomycin-induced lung fibrosis. Int J Mol Med 32: 355-364, 2013.

7. Fang S, Guo H, Cheng Y, Zhou Z, Zhang W, Han B, Luo W, Wang J, Xie W and Chao J: circ HECTD1 promotes the silica-induced pulmonary endothelial-mesenchymal transition via HECTD1. Cell Death Dis 9: 396, 2018.

8. You X, Vlatkovic I, Babic A, Will T, Epstein I, Tushev G, Akbalik G, Wang M, Glock C, Quedenau C, et al: Neural circular RNAs are derived from synaptic genes and regulated by development and plasticity. Nat Neurosci 18: 603-610, 2015.

9. Luo Y, Liu S and Yao K: Transcriptome-wide investigation of mRNA/ circRNA in miR-184 and its r.57c $>$ u mutant type treatment of human lens epithelial cells. Mol Ther Nucleic Acids 7: 71-80, 2017.

10. Zhong Z, Huang M, Lv M, He Y, Duan C, Zhang L and Chen J: Circular RNA MYLK as a competing endogenous RNA promotes bladder cancer progression through modulating VEGFA/VEGFR2 signalling pathway. Cancer Lett 403: 305-317, 2017.

11. Aufiero S, van den Hoogenhof MMG, Reckman YJ, Beqqali A, van der Made I, Kluin J, Khan MAF, Pinto YM and Creemers EE: Cardiac circRNAs arise mainly from constitutive exons rather than alternatively spliced exons. RNA 24: 815-827, 2018.

12. Shi L, Yan P, Liang Y, Sun Y, Shen J, Zhou S, Lin H, Liang X and Cai X: Circular RNA expression is suppressed by androgen receptor (AR)-regulated adenosine deaminase that acts on RNA (ADAR1) in human hepatocellular carcinoma. Cell Death Dis 8: e3171, 2017.

13. Suzuki $\mathrm{H}$ and Tsukahara $\mathrm{T}$ : A view of pre-mRNA splicing from RNase R resistant RNAs. Int J Mol Sci 15: 9331-9342, 2014.

14. Han D, Li J, Wang H, Su X, Hou J, Gu Y, Qian C, Lin Y, Liu X, Huang M, et al: Circular RNA MTO1 acts as the sponge of miR-9 to suppress hepatocelluar carcinoma progression. Hepatology 66: 1151-1164, 2017.
15. Wilusz JE and Sharp PA: Molecular biology. A circuitous route to noncoding RNA. Science 340: 440-441, 2013.

16. Yang C, Yuan W, Yang X, Li P, Wang J, Han J, Tao J, Li P, Yang H, Lv Q and Zhang W: Circular RNA circ-ITCH inhibits bladder cancer progression by sponging $\mathrm{miR}-17 / \mathrm{miR}-224$ and regulating p21, PTEN expression. Mol Cancer 17: 19, 2018.

17. Li F, Zhang L, Li W, Deng J, Zheng J, An M, Lu J and Zhou Y: Circular RNA ITCH has inhibitory effect on ESCC by suppressing the Wnt//-catenin pathway. Oncotarget 6: 6001-6013, 2015.

18. Rybak-Wolf A, Stottmeister C, Glažar P, Jens M, Pino N, Giusti S, Hanan M, Behm M, Bartok O, Ashwal-Fluss R, et al: Circular RNAs in the mammalian brain are highly abundant, conserved, and dynamically expressed. Mol Cell 58: 870-885, 2015.

19. Raghu G, Collard HR, Egan JJ, Martinez FJ, Behr J, Brown KK, Colby TV, Cordier JF, Flaherty KR, Lasky JA, et al: An official ATS/ERS/JRS/ALAT statement: Idiopathic pulmonary fibrosis: Evidence-based guidelines for diagnosis and management. Am J Respir Crit Care Med 183: 788-824, 2011.

20. Chen S, Li T, Zhao Q, Xiao B and Guo J: Using circular RNA hsa_circ_0000190 as a new biomarker in the diagnosis of gastric cancer. Clin Chim Acta 466: 167-171, 2017.

21. Ausubel FM, Brent R, Kingston RE, Moore DD, Seidman JG, Smith JA and Struhl K (eds): Short Protocols in Molecular Biology. 5th edition. Wiley-Blackwell, Hoboken, New Jersey, 2002.

22. Ashwal-Fluss R, Meyer M, Pamudurti NR, Ivanov A, Bartok O, Hanan M, Evantal N, Memczak S, Rajewsky N and Kadener S: circRNA biogenesis competes with pre-mRNA splicing. Mol Cell 56: 55-66, 2014.

23. Ivanov A, Memczak S, Wyler E, Torti F, Porath HT, Orejuela MR, Piechotta M, Levanon EY, Landthaler M, Dieterich C and Rajewsky N: Analysis of Intron sequences reveals hallmarks of circular RNA biogenesis in animals. Cell Rep 10: 170-177, 2015.

24. Burd CE, Jeck WR, Liu Y, Sanoff HK, Wang Z and Sharpless NE: Expression of linear and novel circular forms of an INK4/ARF-associated non-coding RNA correlates with atherosclerosis risk. PloS Genet 6: e1001233, 2010.

25. Zheng Q, Bao C, Guo W, Li S, Chen J, Chen B, Luo Y, Lyu D, Li Y, Shi G, et al: Circular RNA profiling reveals an abundant circHIPK 3 that regulates cell growth by sponging multiple miRNAs. Nat Commun 7: 11215, 2016.

26. Wang $Y$ and Wang Z: Efficient backsplicing produces translatable circular mRNAs. RNA 21: 172-179, 2015.

27. Chao CW, Chan DC, Kuo A and Leder P: The mouse formin (Fmn) gene: Abundant circular RNA transcripts and gene-targeted deletion analysis. Mol Med 4: 614-628, 1998.

28. Li Z, Huang C, Bao C, Chen L, Lin M, Wang X, Zhong G, Yu B, $\mathrm{Hu}$ W, Dai L, et al: Exon-intron circular RNAs regulate transcription in the nucleus. Nat Struct Mol Biol 22: 256-264, 2015.

29. Yang IV, Pedersen BS, Rabinovich E, Hennessy CE, Davidson EJ, Murphy E, Guardela BJ, Tedrow JR, Zhang Y, Singh MK, et al: Relationship of DNA methylation and gene expression in idiopathic pulmonary fibrosis (IPF). Am J Respir Crit Care Med 190: 1263-1272, 2014.

30. André PA, Prêle CM, Vierkotten S, Carnesecchi S, Donati Y, Chambers RC, Pache JC, Crestani B, Barazzone-Argiroffo C, Königshoff M, et al: BARD1 mediates TGF- $\beta$ signaling in pulmonary fibrosis. Respir Res 16: 118, 2015.

31. Suaud L, Miller K, Panichelli AE, Randell RL, Marando CM and Rubenstein RC: 4-Phenylbutyrate stimulates Hsp70 expression through the Elp2 component of elongator and STAT-3 in cystic fibrosis epithelial cells. J Biol Chem 286: 45083-45092, 2011.

32. Link LA, Howley BV, Hussey GS and Howe PH: PCBP1/HNRNP E1 protects chromosomal integrity by translational regulation of CDC27. Mol Cancer Res 14: 634-646, 2016.

33. Aguilar-Martinez E, Chen X, Webber A, Mould AP, Seifert A Hay RT and Sharrocks AD: Screen for multi-SUMO-binding proteins reveals a multi-SIM -binding mechanism for recruitment of the transcriptional regulator ZMYM2 to chromatin. Proc Natl Acad Sci USA 112: E4854-E4863, 2015.

34. Holdt LM, Stahringer A, Sass K, Pichler G, Kulak NA, Wilfert W, Kohlmaier A, Herbst A, Northoff BH, Nicolaou A, et al: Circlar non-coding RNA ANRIL modulates ribosomal RNA maturation and atherosclersis in humans. Nat Commun 7: 12429, 2016.

35. Ghosal S, Das S, Sen R and Chakrabarti J: HumanViCe: Host ceRNA network in virus infected cells in human. Front Genet 5 : $249,2014$.

36. Hansen TB, Jensen TI, Clausen BH, Bramsen JB, Finsen B, Damgaard CK and Kjems J: Natural RNA circles function as efficient microRNA sponges. Nature 495: 384-388, 2013. 
37. Zhang H, Liu X, Chen S, Wu J, Ye X, Xu L, Chen H, Zhang D, Tan R and Wang Y: Tectorigenin inhibits the in vitro proliferation and enhances miR-338 expression of pulmonary fibroblasts in rats with idiopathic pulmonary fibrosis. J Ethnopharmacol 131: $165-173,2010$.

38. Wang L, Ma L, Fan H, Yang Z, Li L and Wang H: MicroRNA-9 regulates cardiac fibrosis by targeting PDGFR- $\beta$ in rats. J Physiol Biochem 72: 213-223, 2016.

39. Wang YS, Li SH, Guo J, Mihic A, Wu J, Sun L, Davis K, Weisel RD and Li RK: Role of miR-145 in cardiac myofibroblast differentiation. J Mol Cell Cardiol 66: 94-105, 2014

40. Rupaimoole R, Ivan C, Yang D, Gharpure KM, Wu SY, Pecot CV, Previs RA, Nagaraja AS, Armaiz-Pena GN, McGuire M, et al: Hypoxia-upregulated microRNA-630 targets Dicer, leading to increased tumor progression. Oncogene 35: 4312-4320, 2016

41. Chen Y, Wang SX, Mu R, Luo X, Liu ZS, Liang B, Zhuo HL, Hao XP, Wang Q, Fang DF, et al: Dysregulation of the miR-324-5p-CUEDC2 axis leads to macrophage dysfunction and is associated with colon cancer. Cell Rep 7: 1982-1993, 2014

42. Kosla J, Dvorakova M, Dvorak M and Cermak V: Effective myofibroblast dedifferentiation by concomitant inhibition of TGF- $\beta$ signaling and perturbation of MAPK signaling. Eur J Cell Biol 92: 363-373, 2013

43. Lam AP, Herazo-Maya JD, Sennello JA, Flozak AS, Russell S, Mutlu GM, Budinger GR, DasGupta R, Varga J, Kaminski N and Gottardi CJ: Wnt coreceptor Lrp5 is a driver of idiopathic pulmonary fibrosis. Am J Respir Crit Care Med 190: 185-195, 2014.

44. Tang CM, Zhang M, Huang L, Hu ZQ, Zhu JN, Xiao Z, Zhang Z, Lin QX, Zheng XL, Yang M, et al: circRNA 000203 enhances the expression of fibrosis-associated genes by derepressing targets of miR-26b-5p, Colla2 and CTGF, in cardiac fibroblats. Sci Rep 7: 40342, 2017

45. Zhou B and Yu JW: A novel identified circular RNA, circRNA 010567, promotes myocardial fibrosis via suppressing miR-141 by targeting TGF- $\beta 1$. Biochem Biophys Res Commun 487: 769-775, 2017.
46. Sui W, Shi Z, Xue W, Ou M, Zhu Y, Chen J, Lin H, Liu F and Dai Y: Circular RNA and gene expression profiles in gastric cancer based on microarray chip technology. Oncol Rep 37: 1804-1814, 2017

47. Zhang Y, Liu H, Li W, Yu J, Li J, Shen Z, Ye G, Qi X and Li G: circRNA_100269 is downregulated in gastric cancer and suppresses tumor cell growth by targeting miR-630. Aging (Albany NY) 9: 1585-1594, 2017.

48. Chen L, Zhang S, Wu J, Cui J, Zhong L, Zeng L and Ge S circRNA_100290 plays a role in oral cancer by functioning as a sponge of the miR-29 family. Oncogene 36: 4551-4561, 2017.

49. Kusko RL, Brothers JF II, Tedrow J, Pandit K, Huleihel L, Perdomo C, Liu G, Juan-Guardela B, Kass D, Zhang S, et al: Integrated genomics reveals convergent transcriptomic networks underlying COPD and IPF. Am J Respir Crit Care Med 194: 948-960, 2016.

50. Li X, Lin Y, Yang X, Wu X and He X: Long noncoding RNA $\mathrm{H} 19$ regulates EZH2 expression by interacting with miR-630 and promotes cell invasion in nasopharyngeal carcinoma. Biochem Biophys Res Commun 473: 913-919, 2016.

51. Knipe RS, Tager AM and Liao JK: The Rho kinases: Critical mediators of multiple profibrotic processes and rational targets for new therapies for pulmonary fibrosis. Pharmacol Rev 67: 103-117, 2015.

52. Cao L, Xie B, Yang X, Liang H, Jiang X, Zhang D, Xue $P$, Chen D and Shao Z: MiR-324-5p suppresses hepatocellular carcinoma cell invasion by counteracting ECM degradation through post-transcriptionally downregulating ETS1 and SP1. PloS One 10: e0133074, 2015. 